\title{
A1180V of Cardiac Sodium Channel Gene (SCN5A): Is It a Risk Factor for Dilated Cardiomyopathy or Just a Common Variant in Han Chinese?
}

\author{
Cheng Shen, ${ }^{1,2}$ Lei Xu, ${ }^{1}$ Zhiyin Yang, ${ }^{3}$ Yunzeng Zou, ${ }^{1,4}$ Kai Hu, ${ }^{1}$ Zheng Fan, ${ }^{5}$ Junbo Ge, ${ }^{1,4}$ \\ and Aijun Sun ${ }^{1,4}$ \\ ${ }^{1}$ Shanghai Institute of Cardiovascular Diseases, Zhongshan Hospital, Fudan University, Shanghai 200032, China \\ ${ }^{2}$ Department of Cardiology, Shandong University, Jinan, Shandong 250012, China \\ ${ }^{3}$ Department of Cardiology, Jining Medical College, Jining, Shandong 272067, China \\ ${ }^{4}$ Institutes of Biomedical Sciences, Fudan University, Shanghai 200032, China \\ ${ }^{5}$ Department of Physiology, The University of Tennessee Health Science Center, Memphis, TN 38163, USA
}

Correspondence should be addressed to Aijun Sun; sun.aijun@zs-hospital.sh.cn

Received 17 June 2013; Accepted 18 September 2013

Academic Editor: Stamatios Theocharis

Copyright (c) 2013 Cheng Shen et al. This is an open access article distributed under the Creative Commons Attribution License, which permits unrestricted use, distribution, and reproduction in any medium, provided the original work is properly cited.

Our previous study of a Chinese family with dilated cardiomyopathy (DCM) suggested that A1180V of the cardiac sodium channel gene (SCN5A) was associated with the disease within this family. According to data deposited in dbSNP, however, A1180V has been found in some small samples of the Asian population. In this study, we followed up the affected pedigree and expanded the investigation of the prevalence of A1180V in 460 unrelated healthy Han Chinese. Besides, we searched and analyzed it in other database as well. During the follow-up period, 1 A1180V carrier's condition deteriorated alot, and another 4 carriers progressed to DCM or atrioventricular block (AVB). We also found that the A1180V was absent among the 460 individuals $(0 \%, 0 / 460)$, and the carrier frequency of A1180V among Chinese was about $0.51 \%$ obtained from the 1000 genome project. In conclusion, our finding suggests that A1180V is a potential risk factor for DCM, and it is extremely rare among Healthy Han Chinese.

\section{Introduction}

Dilated cardiomyopathy (DCM) is the most common form of primary cardiomyopathy and is a leading cause for heart transplantations [1] approximately $30 \%-50 \%$ of affected individuals have a familial form of DCM [2]. To date, at least 40 disease genes have been reported to be associated with familial DCM [3], in which; mutations of SCN5A gene which encodes the alpha subunit of the major sodium channel in the heart have been demonstrated to result in myocardial damage and DCM $[4,5]$. Previously, SCN5A has been implicated in conduction disturbances and several electrophysiological phenotypes $[6,7]$, including atrioventricular block (AVB), long QT syndrome, sick sinus syndrome, sudden infant death syndrome, and atrial fibrillation $[8,9]$. Meanwhile, more and more SCN5A mutations were discovered to be related with DCM, such as A1180V [10], D1275N [11], W156X, R225W [12], and R222Q [13].
We first reported finding A1180V, a single nucleotide mutation, c.3539C > T, that occurred in exon 20 of the SCN5A gene in a three-generation Chinese family with agerelated DCM and progressive AVB in 2008 [10]. In that study, A1180V was detected in all studied patients and in six younger unaffected members of the pedigree. The mutation, however, was not found in 200 healthy Han Chinese controls. Experimental data revealed that A1180V channels exhibited a negative transform of voltage-dependent inactivation of the cardiac sodium channels, which lead to a rate-dependent sodium current reduction, and a moderate increase in late sodium current. Correspondingly, our clinical study revealed QRS widening at high heart rates and QTc prolongation at rest in unaffected carriers. These findings suggest that A1180V is a functional mutation that can contribute to the development of DCM [10].

However, data deposited in the dbSNP database [14] show that Al180V has been found in two small sample 
groups of the Asian population. In the first sample group, A1180V (dbSNP refSNP number, rs41310765) was present in a cohort of 47 Han people (R31HANLA) who were part of the Los Angeles Panel at a carrier frequency of $6.4 \%$. In another sample group (HER_ASIAN-PANEL) of 16 individual Asians selected from the Coriell Cell Repository, genetic sequencing yielded a carrier frequency of $6.2 \%$ (http://www.ncbi.nlm.nih.gov/projects/SNP/snp_ref.cgi?rs =41310765). To date, the data obtained from the dbSNP database show that A1180V is virtually absent in other ethnic groups. Thus, these unpublished data suggest that A1180V might be a common variant in Asians, which is inconsistent with our previous observations.

In order to further evaluate the pathogenic role of A1180V in familial DCM and make sure the frequency of A1180V among Healthy Han Chinese, we followed up the threegeneration Chinese family and expanded the control size to screen for A1180V. Besides, we analyzed its frequency in other database as well.

\section{Materials and Methods}

2.1. Study Population. We followed up all the A1180V carriers and noncarriers of the three-generation Chinese family for 6 years. All individuals were interviewed with the symptoms of cardiovascular disease, such as typical chest pain, palpitation, cyanosis, and syncope. 12-lead electrocardiogram (ECG) and echocardiography were performed on the family members to make a diagnosis.

460 unrelated Healthy Han Chinese living in Shanghai, ranging from 20 to 70 years old, were enrolled to the control group from a National High Technology Research and Development Program (the National 863 Program) of China. Individuals with known cardiovascular risk factors other than age, gender, and smoking history were excluded. The enrolled participants did not show any abnormalities under comprehensive clinical evaluation, including review of medical history, physical examination, 12-lead ECG, submaximal exercise ECG, and blood biochemical examinations.

Each of the participants and individuals whose data are included here signed an informed consent form after a complete and clear explanation of the nature and purpose of the study. The study was approved by the local Ethics Committee of Zhongshan Hospital, Fudan University.

2.2. Genotype Determination. DNA was extracted from $2 \mathrm{~mL}$ venous blood according to kit procedure (Sigma, USA) and stored at $-80^{\circ} \mathrm{C}$. The exon 20 of SCN5A was amplified by polymerase chain reaction (PCR). A $20 \mathrm{uL}$ mixture was prepared for each reaction and included $1 \mathrm{x}$ HotStarTaq buffer, $2.0 \mathrm{mM} \mathrm{Mg}^{2+}, 0.2 \mathrm{mM}$ dNTP, $0.2 \mu \mathrm{M}$ of each primer, $1 \mathrm{U}$ HotStarTaq polymerase (Qiagen Inc.) and $1 \mu \mathrm{L}$ template DNA. The DNA was amplified by cycling at $95^{\circ} \mathrm{C}$ for $15 \mathrm{~min}$; 11 cycles of $94^{\circ} \mathrm{C}$ for $15 \mathrm{~s}, 62^{\circ} \mathrm{C}-0.5^{\circ} \mathrm{C}$ per cycle for $40 \mathrm{~s}, 72^{\circ} \mathrm{C}$ for $1 \mathrm{mins}$; 24 cycles of $94^{\circ} \mathrm{C}$ for $15 \mathrm{~s}, 57^{\circ} \mathrm{C}$ for $30 \mathrm{~s}, 72^{\circ} \mathrm{C}$ for $1 \mathrm{mins} ; 72^{\circ} \mathrm{C}$ for $2 \mathrm{~min}$. The oligonucleotide sequences of the primers were as follows: $5^{\prime}$-ACCCCCATCATCGTAGCTCTT- $3^{\prime}$ and $5^{\prime}$ TCCTGCTCTGGCCTCCATAC-3'. The PCR products were purified by $1 \mathrm{U}$ SAP and $6 \mathrm{U}$ Exo I, and they were added into $8 \mu \mathrm{L}$ PCR products. The mixture was incubated at $37^{\circ} \mathrm{C}$ for $60 \mathrm{mins}$, followed by incubation at $70^{\circ} \mathrm{C}$ for $10 \mathrm{mins}$. Reaction mixture included $2 \mu \mathrm{L}$ BigDye $3.1 \mathrm{mix}, 2 \mu \mathrm{L}$ sequencing primer $\left(0.4 \mu \mathrm{M}, 5^{\prime}\right.$-ACCCCCATCATCGTAGCTCTT3 $\left.{ }^{\prime}\right)$ and $1-2 \mu \mathrm{L}$ purified PCR product. The cycling program was $96^{\circ} \mathrm{C}$ for $1 \mathrm{~min} ; 28$ cycles of $96^{\circ} \mathrm{C}$ for $10 \mathrm{~s}, 50^{\circ} \mathrm{C}$ for $5 \mathrm{~s}, 60^{\circ} \mathrm{C}$ for 4 mins. The sequencing products were analyzed by an ABI3730XL DNA Analyzer. The results were analyzed by the Polyphred software and revised manually.

2.3. Database Retrieval. We searched A1180V of SCN5A in 1000 genomes project [15]. Besides, we have excluded some familial data to make sure that the frequency of A1180V from 1000 genomes project was calculated from unrelated individuals.

2.4. Statistical Analyses. All statistical analyses were performed with the SAS statistical program. Comparison of the A1180V carrier frequency between our studies and the results of dbSNP database was tested by using the Fisher's exact test. Statistical significance was determined at $P<0.05$.

\section{Results}

Combined with our former study and the 6 years of clinical followup of the affected pedigree, we discovered that A1180V related DCM was preceded by progressive AVB which mostly occurred in the third decade of life. During the follow-up period, the condition of an A1180V carrier (II-8 in Figure 1) who had been diagnosed with DCM before aggravated gradually and she now was diagnosed with severe congestive heart failure. Besides, III-1 with A1180V, developed to DCM and complete atrioventricular block (AVB) and another 3 carriers (III-2, III-3, III-13) progressed to different degree of AVB. Figure 2 displays changes of echocardiograms of III-1 and ECG recordings of III-2 in the follow-up period. None of the individuals in the pedigree showed any sign of long-QT or Brugada syndrome and other diseases.

After screening A1180V in 460 individuals, we found that A1180V was absent in this group (0/460, 0\%). Figure 3(a) gives an example of the sequencing results, and Figure $3(\mathrm{~b})$ is a sequencing result of a A1180V carrier in 2007. The combined carrier frequency, $0 \%$ (i.e., 0 out of 660 cases), of A1180V from our studies remarkably suggests that $\mathrm{A} 1180 \mathrm{~V}$ is not a common variant among Healthy Chinese, and the data significantly differs from that in the dbSNP database $\left(P=5.3 \times 10^{-5}\right)$.

Data from the 1000 genomes project revealed a carrier frequency of $0.51 \%$ (i.e., 1 out of 197 cases) for A1180V among Chinese participants. The data was obtained by analyzing the sequencing results of 97 northern and 100 southern Chinese adults. Since the southern Chinese group was familial related, the data from known offspring were excluded from the calculation of the carrier frequency to make sure the value was calculated from unrelated individuals (http://browser .1000genomes.org/Homo_sapiens/Variation/Population?db= core; $\mathrm{r}=3: 38616415-38617415 ; \mathrm{v}=\mathrm{rs} 41310765 ; \mathrm{vdb}=$ variation; $\mathrm{vf}=$ 12532571). The data also dramatically differs from those 


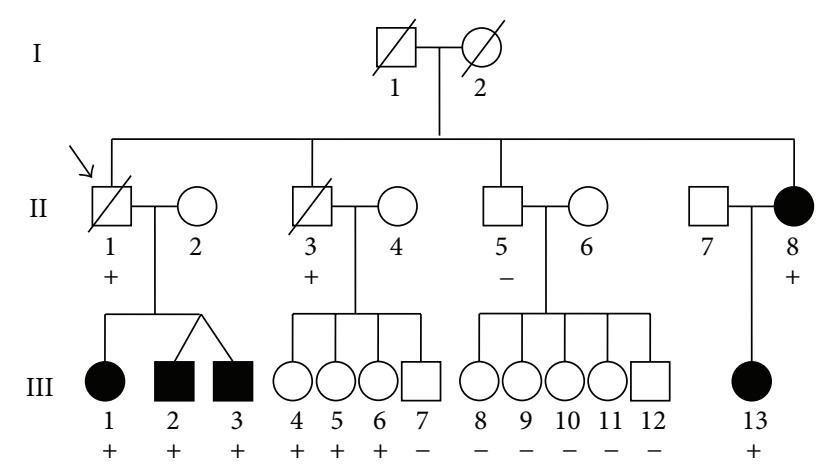

FIGURE 1: Pedigree of the affected family. This figure mostly indicates the results of the 6 years followup, and the former characteristics of this family could be obtained from our published data in 2008 [10]. Individuals of the affected family are represented by squares (males) and circles (females). Five carriers whose disease have progressed to severe DCM or AVB during the follow-up period are marked by darkened symbols. The slash indicates the decreased people, and the arrow represents the proband. A1180V carriers and noncarriers are represented by “+" and "-", respectively.

deposited in the dbSNP database $(P=0.013)$. Table 1 summarizes the results of different studies of the carrier frequency of A1180V.

\section{Discussion}

We have presented the followup and sequencing data identifying that A1180V of SCN5A was a potential risk factor for DCM, and it is absent among healthy Han Chinese. Compared with the former results in 2008, 5 A1180V carriers exhibited age-related progressive AVB and DCM, and the A1180V carrier frequency among Han Chinese in our study is $0 \%$ out of 660 control cases.

Evidence is now being accumulated that SCN5A mutations are associated with development of cardiac dilation [13, 16-18]. McNair et al. have reported that mutations in SCN5A were detected in $1.7 \%$ of DCM families with a shared mechanism of disruption of the voltage-sensing mechanism of this channel leading to DCM [19]. Moreover, some SCN5A mutations which have been found to be a primary factor of leading DCM were characterized by an age-related phenotype of cardiac dilation with different types of arrhythmia [11, 20]. And the pathogenic characteristic of Al180V conformed to the pattern as well. Since the cardiac sodium channel is principally in charge of the fast depolarization of the myocardium [21], some suggested that the cardiac dilation may result from long-term arrhythmia [22, 23]. However, our experimental evidence indicated that A1180V could alter calcium homeostasis as a consequence of alterations in intracellular sodium concentrations, and the former condition might damage myocardium directly and subsequently lead to cardiac dilation [10].

Our data have revealed that A1180V specifically appeared in the affected pedigree and was absent among healthy controls, however, why do such significant discrepancies exist for A1180V among different studies of the same ethnic population? Possible explanations for the discrepancies
TABLE 1: Different studies of the distribution of A1180V among Chinese.

\begin{tabular}{lccc}
\hline & Individuals; $n$ & Al180V carrier; $n$ & Carrier frequency \\
\hline dbSNP $^{\mathrm{a}}$ & 63 & 4 & $6.3 \%$ \\
1000 genomes & 197 & 1 & $0.51 \%^{*}$ \\
Our study & 660 & 0 & $0 \%{ }^{*}$
\end{tabular}

${ }^{\mathrm{a}}$ Data from the dbSNP including 47 Han people and 16 individual Asians.

${ }^{*} P=0.013$ versus dbSNP.

${ }^{\#} P=5.3 \times 10^{-5}$ versus dbSNP.

include the following. (1) Different Asian ethnic subgroups may have different genetic backgrounds that cause differing prevalence of a genetic variant. For example, R1193Q (another SCN5A variant) is more prevalent in Asians living in Taiwan (frequency 12\%) compared to Asians living in Japan ( 2\%) [24]. Most Chinese-Americans are descendants of people from southern China, so they may have a genetic background that differs slightly from the majority of the subjects enrolled in our studies. The population of the Shanghai area consists mostly of people who have immigrated from other areas of China. (2) Relatively small sample sizes may also account for high carrier frequency of those data deposited in the dbSNP database. (3) The sequencing depth of different sequencing technologies varies greatly within and between experiments, and it may introduce bias as well [25].

At present, lots of gene mutations were found playing pathogenic roles in both familial and sporadic diseases [26, 27], then another question is that whether A1180V would be a risk factor among sporadic DCM patients as well. In our recent studies of the distributions of candidate mutations in DCM-related genes in sporadic DCM patients conducted using high-throughput sequencing technology, we identified A1180V in 1 out of 68 patients with DCM. The patient, a 41year-old male, was diagnosed as having DCM with NYHA class I, LVEDD $78 \mathrm{~mm}$, and LVEF 24\%, but no other diseases. However, the patient carrying A1180V also carries other mutations in several DCM-related genes, including G1117S, P1119R, Y498H of LAMA4, W768S of RBM20, P398S of VCL, and R659W of SCN5A. The last two mutations have never been reported previously. Although we found that A1180V might also be a risk factor in sporadic DCM patients, we cannot exclude the possibility that A1180V could work together with other gene mutations.

In addition to genetic factors, our previous study of the DCM pedigree carrying A1180V demonstrated that the A1180V channel lost current only at high heart rates, which suggested that physical activity and lifestyle that increased average daily heart rate might enhance the phenotype for A1180V carriers [10]. Therefore, environmental factors may also play a role in expression of the pathogenic mechanism of A1180V. Further studies of genetic and environmental factors and followup of A1180V carriers will provide more information about the relationship between the mutation and the risk of DCM. Studies of transgenic mice may also provide a step to test the hypothesis and to explore the underlying mechanism. 


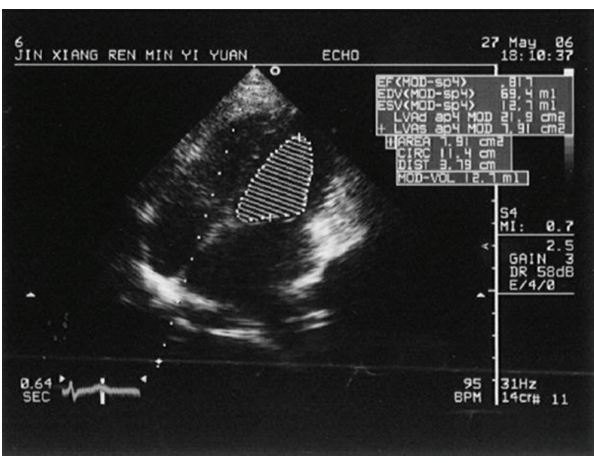

(a)

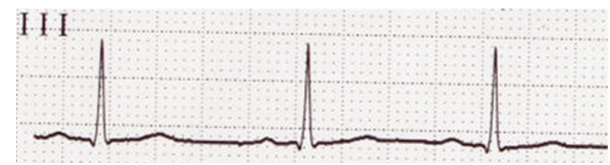

(c)

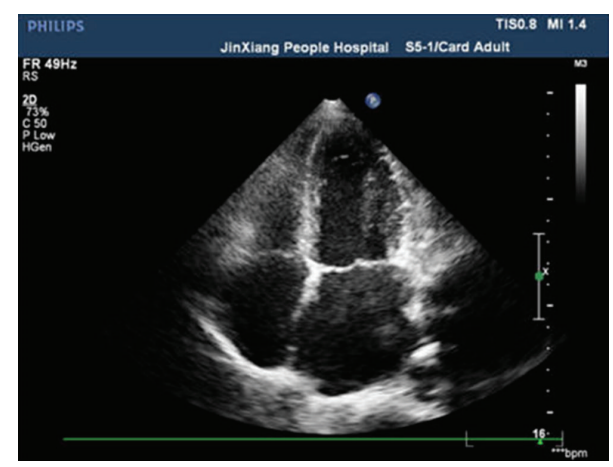

(b)

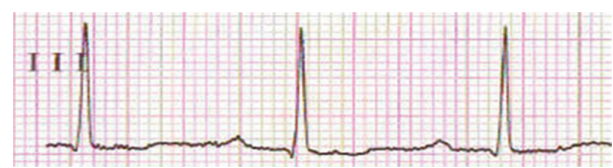

(d)

FIgURE 2: Clinical characteristics of progressive DCM and AVB of the A1180V carriers. (a) and (b) Echocardiograms of III-1. No sign of cardiac dilation was present at age 36 (a), and the patient has progressed to DCM at age 42 (b); (c) and (d) ECG recordings of III-2. ECG showing any abnormalities at age 27 (c), and the patient was diagnosed with first-degree AVB after 6 years (d).

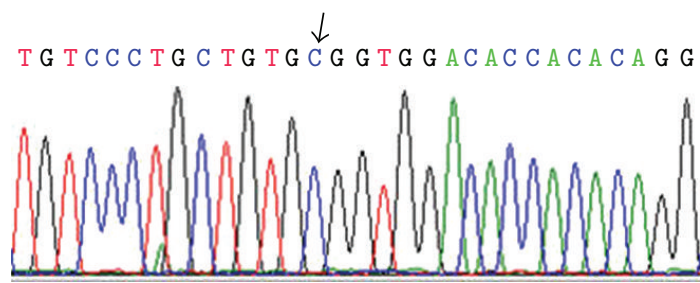

(a)

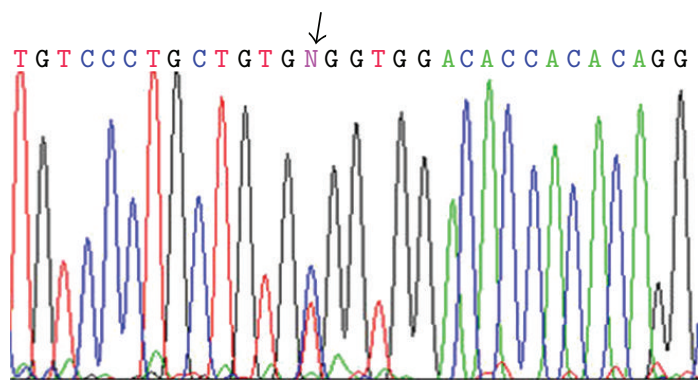

(b)

FIGURE 3: Results of genomic DNA sequencing analysis contrast controls and A1180V carriers. (a) Sequencing data obtained from one of the 460 control individuals does not show a heterozygous nucleotide at 1180 in exon 20 of SCN5A; (b) sequencing data from a A1180V carrier obtained in previous data. Arrows indicate the position of c.3539C > T (A1180V).

\section{Conclusions}

In conclusion, herein we provide evidence that the A1180V is a potential cause for DCM rather than a common variant in Han Chinese, although additional genetic or environmental factors may potentiate the risk for DCM in A1180V carriers.

\section{Conflict of Interests}

The authors declare that there is no conflict of interests regarding the publication of this paper.

\section{Author's Contribution}

Cheng Shen and Lei Xu contributed equally to this work.

\section{Acknowledgments}

The authors appreciate the individuals and clinicians who have submitted clinical samples or offered medicine help. Also thank for the sequencing services from the Center of Genetic and Genomic Analysis, Genesky Biotechnologies Inc., Shanghai. This study is supported by the Grant (81170202) to Aijun Sun from the Surface Project of the National Natural Science Foundation of China (NSFC) and the Grant (09SG03) to Aijun Sun from the Dawn Plan of Shanghai Education Committee.

\section{References}

[1] B. J. Maron, J. A. Towbin, G. Thiene et al., "Contemporary definitions and classification of the cardiomyopathies: an American Heart Association Scientific Statement from the Council on Clinical Cardiology, Heart Failure and Transplantation Committee; Quality of Care and Outcomes Research and Functional Genomics and Translational Biology Interdisciplinary Working Groups; and Council on Epidemiology and Prevention," Circulation, vol. 113, no. 14, pp. 1807-1816, 2006.

[2] R. Roncarati, C. V. Anselmi, P. Krawitz et al., "Doubly heterozygous LMNA and TTN mutations revealed by exome sequencing in a severe form of dilated cardiomyopathy," European Journal of Human Genetics, vol. 21, no. 10, pp. 1105-11011, 2013. 
[3] D. Fatkin, R. Otway, and Z. Richmond, "Genetics of dilated cardiomyopathy," Heart Failure Clinics, vol. 6, no. 2, pp. 129-140, 2010.

[4] E. Schulze-Bahr, "Cardiac sodium channels: dysregulation meets myocardial failure," Cardiovascular Research, vol. 75, no. 3, pp. 455-456, 2007.

[5] T. M. Olson, V. V. Michels, J. D. Ballew et al., "Sodium channel mutations and susceptibility to heart failure and atrial fibrillation," Journal of the American Medical Association, vol. 293, no. 4, pp. 447-454, 2005.

[6] A. A. M. Wilde and C. R. Bezzina, "Genetics of cardiac arrhythmias," Heart, vol. 91, no. 10, pp. 1352-1358, 2005.

[7] A. L. George Jr., "Inherited disorders of voltage-gated sodium channels," Journal of Clinical Investigation, vol. 115, no. 8, pp. 1990-1999, 2005.

[8] A. A. M. Wilde and R. Brugada, "Phenotypical manifestations of mutations in the genes encoding subunits of the cardiac sodium channel," Circulation Research, vol. 108, no. 7, pp. 884-897, 2011.

[9] D. Darbar, P. J. Kannankeril, B. S. Donahue et al., "Cardiac sodium channel (SCN5A) variants associated with atrial fibrillation," Circulation, vol. 117, no. 15, pp. 1927-1935, 2008.

[10] J. Ge, A. Sun, V. Paajanen et al., "Molecular and clinical characterization of a novel SCN5A mutation associated with atrioventricular block and dilated cardiomyopathy," Circulation, vol. 1, no. 2, pp. 83-92, 2008.

[11] W. P. McNair, L. Ku, M. R. G. Taylor et al., "SCN5A mutation associated with dilated cardiomyopathy, conduction disorder, and arrhythmia," Circulation, vol. 110, no. 15, pp. 2163-2167, 2004.

[12] C. R. Bezzina, M. B. Rook, W. A. Groenewegen et al., "Compound heterozygosity for mutations (W156X and R225W) in SCN5A associated with severe cardiac conduction disturbances and degenerative changes in the conduction system," Circulation Research, vol. 92, no. 2, pp. 159-168, 2003.

[13] S. A. Mann, M. L. Castro, M. Ohanian et al. et al., "R222Q SCN5A mutation is associated with reversible ventricular ectopy and dilated cardiomyopathy," American College of Cardiology, vol. 60, no. 16, pp. 1566-1573, 2012.

[14] S. T. Sherry, M. H. Ward, M. Kholodov et al., "dbSNP: the NCBI database of genetic variation," Nucleic Acids Research, vol. 29, no. 1, pp. 308-311, 2001.

[15] G. R. Abecasis, D. Altshuler, A. Auton et al. et al., "A map of human genome variation from population-scale sequencing," Nature, vol. 467, no. 7319, pp. 1061-1073, 2010.

[16] K. Y. van Spaendonck-Zwarts, I. A. van Rijsingen, M. P. van den Berg et al., "Genetic analysis in 418 index patients with idiopathic dilated cardiomyopathy: overview of 10 years' experience," European Journal of Heart Failure, vol. 15, no. 6, pp. 628-636, 2013.

[17] P. Gosselin-Badaroudine, I. D. Keller, I. D. Hai Huang et al., "A proton leak current through the cardiac sodium channel is linked to mixed arrhythmia and the dilated cardiomyopathy phenotype," PLoS One, vol. 7, no. 5, Article ID e38331, 2012.

[18] E. Rampersaud, J. D. Siegfried, N. Norton, D. Li, E. Martin, and R. E. Hershberger, "Rare variant mutations identified in pediatric patients with dilated cardiomyopathy," Progress in Pediatric Cardiology, vol. 31, no. 1, pp. 39-47, 2011.

[19] W. P. McNair, G. Sinagra, M. R. Taylor et al., "SCN5A mutations associate with arrhythmic dilated cardiomyopathy and commonly localize to the voltage-sensing mechanism," American College of Cardiology, vol. 57, no. 21, pp. 2160-2168, 2011.
[20] R. Shi, Y. Zhang, C. Yang et al., "The cardiac sodium channel mutation delQKP, 1507-1509 is associated with the expanding phenotypic spectrum of LQT3, conduction disorder, dilated cardiomyopathy, and high incidence of youth sudden death," Europace, vol. 10, no. 11, pp. 1329-1335, 2008.

[21] Y. Ruan, N. Liu, and S. G. Priori, "Sodium channel mutations and arrhythmias," Nature reviews. Cardiology, vol. 6, no. 5, pp. 337-348, 2009.

[22] W. A. Groenewegen and A. A. Wilde, "Letter regarding article by McNair et al, 'SCN5A mutation associated with dilated cardiomyopathy, conduction disorder, and arrhythmia,' Circulation, vol. 112, no. 1, pp. e9-e10, 2005.

[23] C. R. Bezzina and C. A. Remme, "Dilated cardiomyopathy due to sodium channel dysfunction: what is the connection?" Circulation, vol. 1, no. 2, pp. 80-82, 2008.

[24] H. W. Hwang, J. J. Chen, Y. J. Lin et al., "R1193Q of SCN5A, a Brugada and long QT mutation, is a common polymorphism in Han Chinese," Journal of Medical Genetics, vol. 42, no. 2, article e7, 2005.

[25] C. Garner, "Confounded by sequencing depth in association studies of rare alleles," Genetic Epidemiology, vol. 35, no. 4, pp. 261-268, 2011.

[26] N. Norton, D. Li, E. Rampersaud et al., "Exome sequencing and genome-wide linkage analysis in 17 families illustrate the complex contribution of TTN truncating variants to dilated cardiomyopathy," Circulation, vol. 6, no. 2, pp. 144-153, 2013.

[27] H. Kabata, T. Satoh, M. Kataoka et al., "BMPR2 mutations, clinical phenotypes and outcomes of Japanese patients with sporadic or familial pulmonary hypertension," Respirology, vol. 18, no. 7, pp. 1076-1082, 2013. 


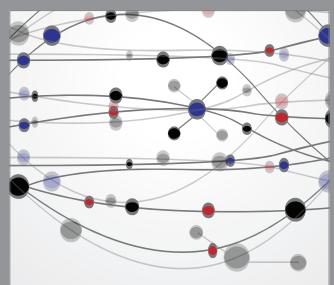

The Scientific World Journal
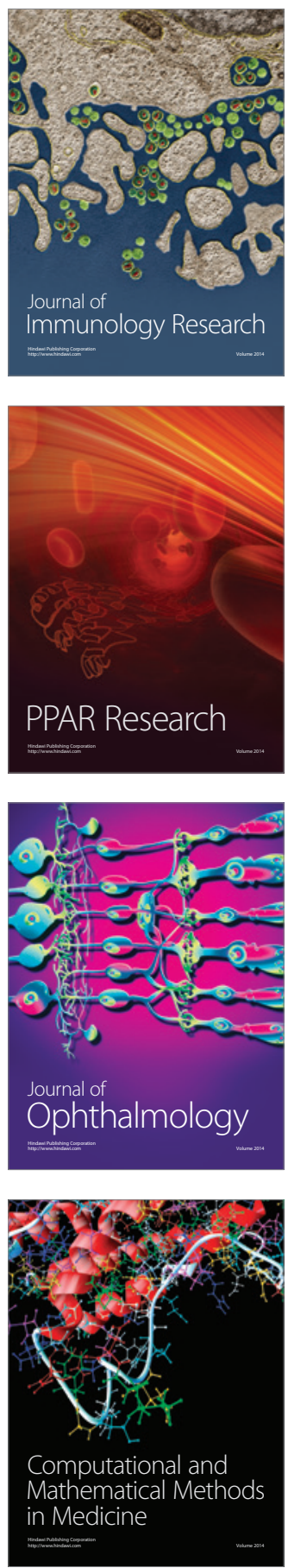

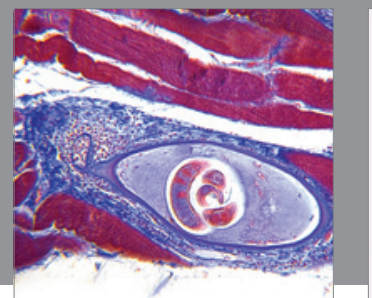

Gastroenterology

Research and Practice
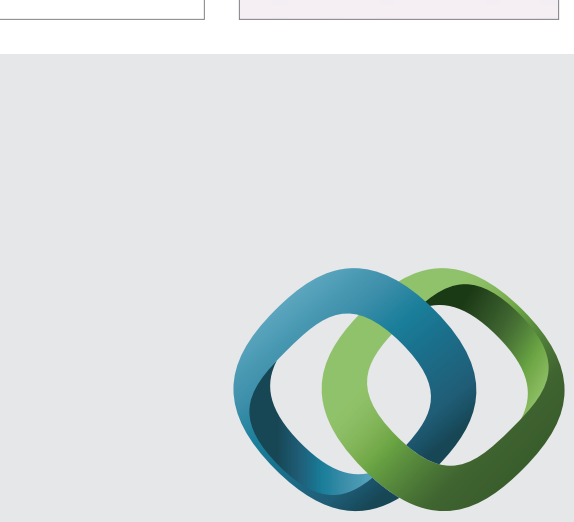

\section{Hindawi}

Submit your manuscripts at

http://www.hindawi.com
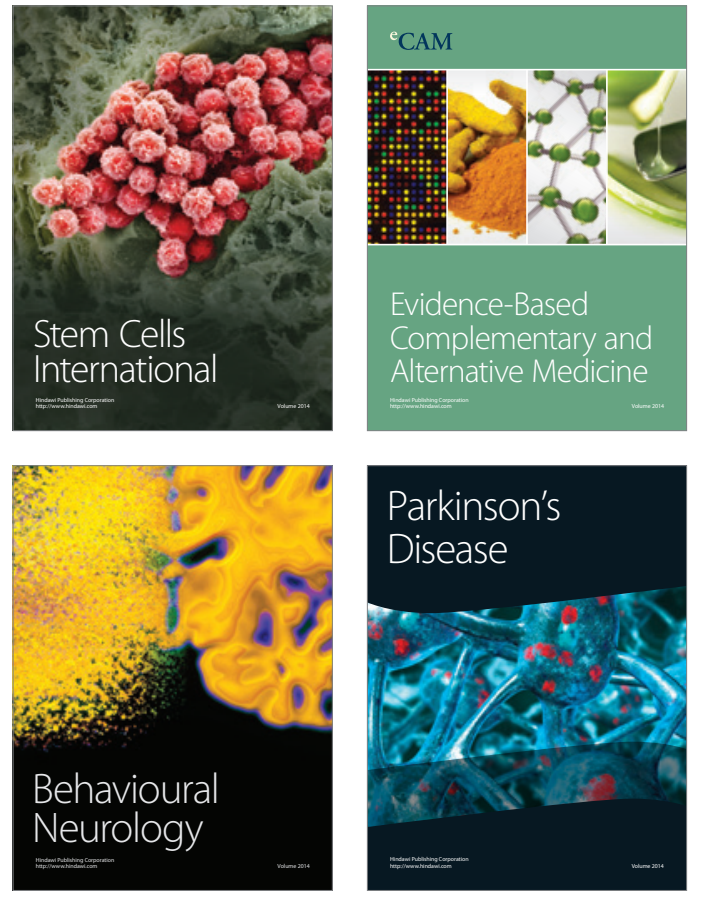
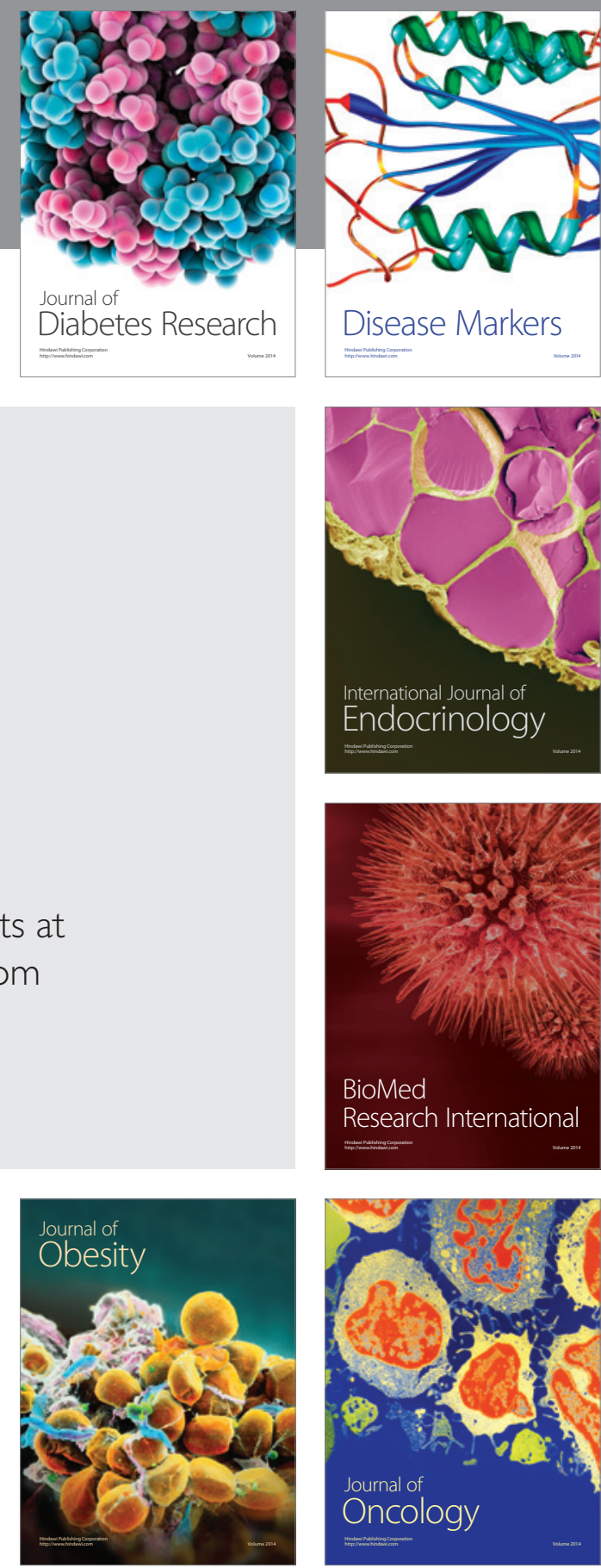

Disease Markers
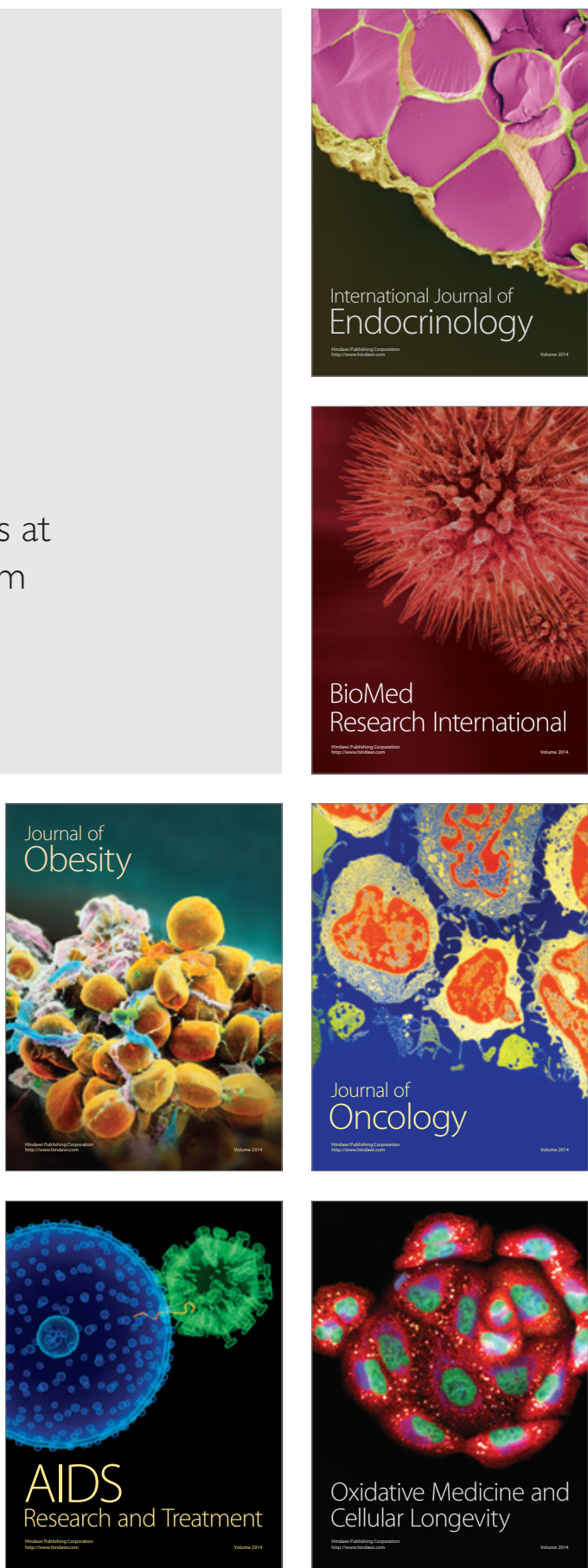